\title{
Production Inventory System under Weibull Amelioration, Pareto Deterioration and Exponentially Time Based Demand under Fully Backlogged Shortages
}

\author{
Ankit Bhojak \\ Assistant Professor \\ Department of Statistics, \\ GLS Institute of Commerce, \\ GLS University, Ahmedabad, India \\ bhojak.ankit@gmail.com
}

\author{
U. B. Gothi \\ Head and Associate Professor \\ Department of Statistics, \\ St. Xavier's College (Autonomous), \\ Ahmedabad, India \\ ubgothi@gmail.com
}

\begin{abstract}
In this research paper we have considered production inventory model for the items whose demand starts at the same time with its production. In the initial phase of production, we have considered time to ameliorate following two parameter Weibull distribution and demand is an exponential function of time. After some period effect of deterioration can be observed, so in the second phase, we have assumed deterioration of items following Pareto type - I distribution with the same amelioration rate and demand rate as in previous time interval. Here shortages are allowed to occur and unsatisfied demand is fully backlogged. The model so derived is illustrated with a numerical example with its sensitivity analysis.
\end{abstract}

Keywords: Weibull distribution, Pareto type - I distribution, Fully backlogged shortage

\section{INTRODUCTION}

Various inventory models have been developed by researchers in last few decades. A note on the EPQ model with shortages and variable lead time was given by Chang [3]. Lan, Yu, Lin, Tung, Yen and Deng [4] had presented a note on the improved algebric method for the EPQ model with stochastic lead time. Shamsi, Haji, Shadrokh and Nourbakhsh [13] published their work on economic production quantity in reworkable production systems with inspection errors, scraps and backlogging. Wee, Wang and Yang [5] had formulated a production quantity model for imperfect quality items with shortage and screening constraint. Moreover Gothi and Chatterji [22] have developed EPQ model for imperfect quality items under constant demand rate and varying IHC. Li, Lan and Mawhinney [15] reviewed on deteriorating inventory study. Economic production quantity models with shortage, price and stock-dependent demand for deteriorating items were developed by Jain, Sharma and Rathore [11]. Bansal and Ahalawat [8] have given integrated inventory models for decaying items with exponential demand under inflation. An EPQ model using Weibull distributed deterioration item with time varying holding cost was formulated by Kawale and Bansode [9]. Bhojak and Gothi [1] have developed EPQ model with time dependent IHC and Weibull distributed deterioration under shortages.

In addition to this, in order to make inventory models more appropriate for the current market scenario, amelioration of items is also considered along with its deterioration. A stochastic setcovering location model for both ameliorating and deteriorating items was given by Hwang [6]. Law and Wee [16] had also presented an integrated production inventory for ameliorating and deteriorating items taking account of time discounting. Optimal control of an inventory system with variable demand and ameliorating / deteriorating items was considered by Srichandan Mishra, Raju, U.K.Misra and G.Misra [19]. Bhojak and Gothi [2] have formulated two inventory models for ameliorating and deteriorating items with time dependent demand and IHC. Further an integrated inventory model with exponential amelioration and two parameter Weibull deterioration was developed by Gothi, Chatterji and Parmar [20].

Rao, Begum and Murty [14] had given optimal ordering policies of inventory model for deteriorating items having generalized Pareto lifetime. Singh, Vaish and Singh, S. R. [18] had presented an EOQ 
model with Pareto distribution for deterioration, trapezoidal type demand and backlogging under trade credit policy. Inventory model for deteriorating items having two component mixture of Pareto lifetime and selling price dependent demand was formulated by Vijayalakshmi. G, Srinivasa Rao. K and Nirupama Devi [23]. Gothi and Bhojak [21] .

In this paper, we have developed a production inventory model by considering two parameter Weibull distributed amelioration till stock exists. The distribution of the time to deteriorate is a random variable following two parameter Pareto type - I distribution. The probability density function of two parameter Pareto type - I distribution, given by $\mathrm{f}(\mathrm{t})=\frac{\theta}{\mu}\left(\frac{\mathrm{t}}{\mu}\right)^{-\theta-1} ; \mathrm{t} \geq \mu$, where $\theta$ and $\mu$ are parameters with positive real value. The instantaneous rate of deterioration $\theta(\mathrm{t})$ of the non-deteriorated inventory at time $\mathrm{t}$, can be obtained from $\theta(\mathrm{t})=\frac{\mathrm{f}(\mathrm{t})}{1-\mathrm{F}(\mathrm{t})}$, where $\mathrm{F}(\mathrm{t})=1-\left(\frac{\mathrm{t}}{\mu}\right)^{-\theta}$ is the cumulative distribution function for the two parameter Pareto type - I distribution. Thus, the instantaneous rate of deterioration of the on-hand inventory is $\theta(\mathrm{t})=\frac{\theta}{\mathrm{t}}$ and demand rate is an exponential function of time. In this model shortages are allowed to occur and unsatisfied demand is fully backlogged.

\section{ASSUMPTIONS}

The following assumptions are considered to develop this model

1. The inventory system involves only one item and one stocking point.

2. Replenishment rate is infinite.

3. Lead-time is zero.

4. The amelioration occurs when the item is effectively in stock but the deterioration starts at time $\mathrm{t}=\mu$

5. The deteriorated items are not replaced during the given cycle.

6. Infinite time horizon period is considered.

7. Shortages are allowed and the unsatisfied demand is fully backlogged.

8. Holding cost $\mathrm{C}_{\mathrm{h}}=\mathrm{h}+\mathrm{rt} \quad(\mathrm{h}, \mathrm{r}>0)$ is a linear function of time.

9. Time to deterioration follows Pareto type - I distribution in the time interval $\left[\mu, t_{1}\right]$ and $\theta(t)=\frac{\theta}{t}$ is a deterioration rate.

10. Demand rate is an exponential type and it is $\mathrm{R}(\mathrm{t})=\lambda \mathrm{t}^{-\mathrm{p}}$ over a time period $[0, \mathrm{~T}]$, where $\lambda$ is a positive constant.

11. The amelioration rate is derived from Weibull distribution with two parameters and it is

$$
\mathrm{A}(\mathrm{t})=\alpha \beta \mathrm{t}^{\beta-1} \quad ; 0 \leq \mathrm{t} \leq \mathrm{t}_{1}
$$

where $\alpha$ and $\beta$ are the positive parameters.

12. Unit amelioration cost, deterioration cost, production cost, ordering cost and shortage cost per unit are known and constants.

13. Total inventory cost is a continuous real function which is convex to the origin.

\section{Notations}

The following notations are used to develop the mathematical model:

1. $\mathrm{Q}(\mathrm{t})$ : Inventory level of the product at time $\mathrm{t}$

2. $\mathrm{R}(\mathrm{t}) \quad$ : Demand rate varying over time. 

3. $A(t):$ Amelioration rate at any time $t$.
4. $\theta(\mathrm{t}):$ Deterioration rate.
5. A : Ordering cost per order during the cycle period.
6. $\mathrm{K}:$ Production rate.
7. $\mathrm{C}_{\mathrm{h}}:$ Inventory holding cost per unit per unit time.
8. $\quad \mathrm{C}_{\mathrm{a}} \quad$ : Amelioration cost per unit.
9. $\mathrm{C}_{\mathrm{d}} \quad$ : Deterioration cost per unit.
10. $\mathrm{C}_{\mathrm{p}} \quad$ : $\quad$ Production cost per unit. $\left(\mathrm{C}_{\mathrm{p}}>\mathrm{C}_{\mathrm{a}}\right)$
11. $\mathrm{C}_{\mathrm{s}}:$ Shortage cost per unit.
12. $\mathrm{S}_{1} \quad$ : Inventory level at time $\mathrm{t}=\mu$.
13. $\mathrm{S}_{2}:$ The maximum inventory level during shortage period.
14. $\mathrm{T}:$ Duration of a cycle.
15. TC : Total cost per unit time.

\section{MATHEMATICAL Formulation AND SOlUtion}

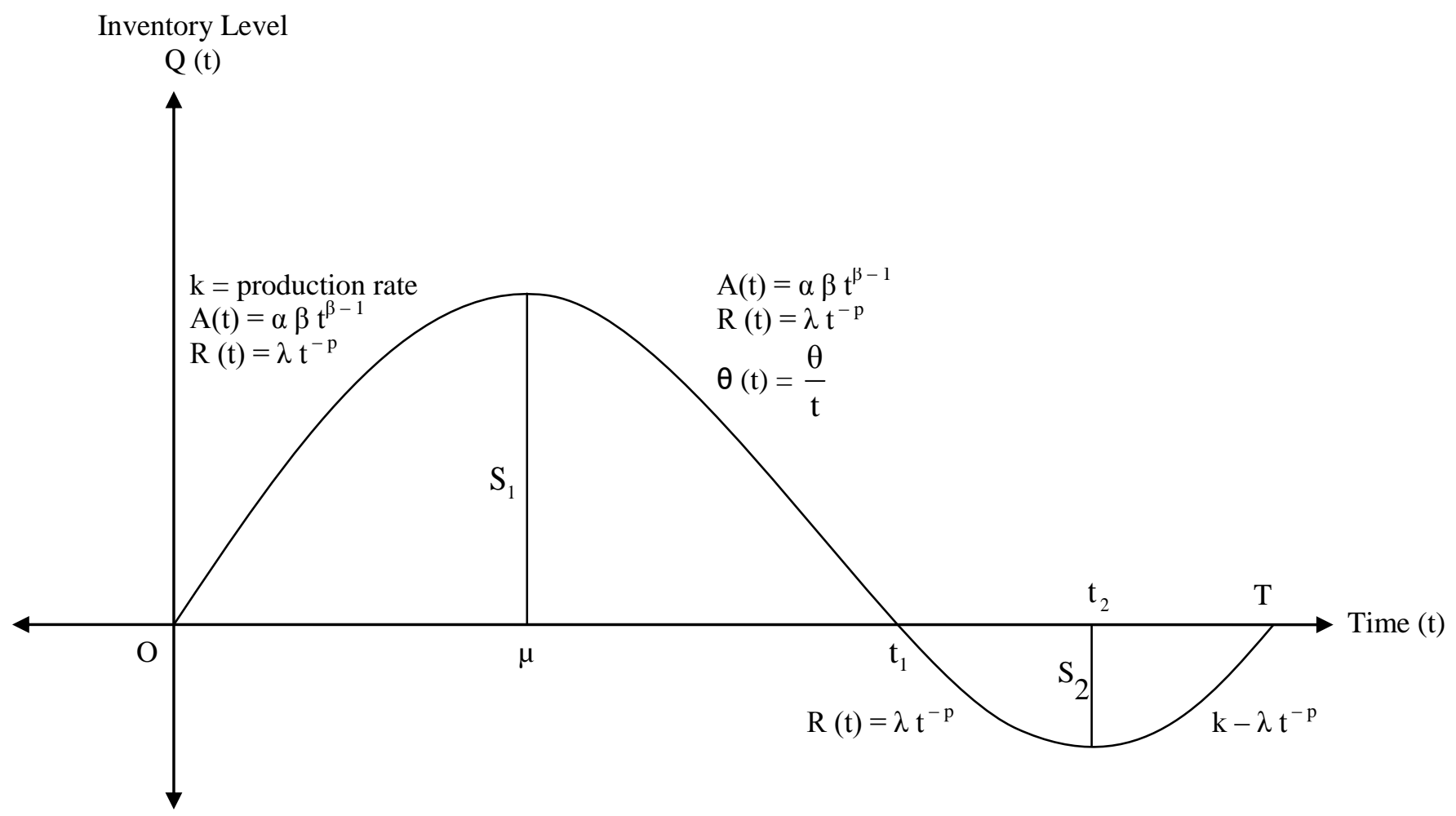

Fig. 1. Graphical presentation of inventory system

At the initial stage, inventory is zero. At time $\mathrm{t}=0$, the production and supply both start simultaneously. Then the production stops at $t=\mu$ where the maximum inventory level $S_{1}$ is reached. During the interval $[0, \mu]$ the inventory is built up at a rate $\mathrm{k}-\lambda \mathrm{t}^{-\mathrm{p}}+\alpha \beta \mathrm{t}^{\beta-1}$. Even though two parameter Weibull amelioration rate exists in time interval $\left[\mu, \mathrm{t}_{1}\right]$, the stock reaches to zero level at time $\mathrm{t}=\mathrm{t}_{1}$ due to demand rate $-\lambda \mathrm{t}^{-\mathrm{p}}$ along with the Pareto type $-\mathrm{I}$ deterioration rate $\frac{\theta}{\mathrm{t}}$. Thereafter, shortages are allowed to occur during the time interval $\left[\mathrm{t}_{1}, \mathrm{t}_{2}\right]$ at a rate $-\lambda \mathrm{t}^{-\mathrm{p}}$. At time $\mathrm{t}=\mathrm{t}_{2}$ shortage reaches at the maximum level $\mathrm{S}_{2}$ and then production starts at the same rate and the backlog is fulfilled at a rate of $\mathrm{k}-\lambda \mathrm{t}^{-\mathrm{p}}$ in the time interval $\left[\mathrm{t}_{2}, \mathrm{~T}\right]$. The stock level becomes zero at time $\mathrm{t}=\mathrm{T}$. The same cycle is repeated for the further time period $\mathrm{T}$. 
Differential Equations pertaining to the situations as explained above are given by

$$
\begin{array}{ll}
\frac{\mathrm{dQ}(\mathrm{t})}{\mathrm{d} \mathrm{t}}=\alpha \beta \mathrm{t}^{\beta-1} \mathrm{Q}(\mathrm{t})+\mathrm{k}-\lambda \mathrm{t}^{-\mathrm{p}} & 0 \leq \mathrm{t} \leq \mu \\
\frac{\mathrm{dQ}(\mathrm{t})}{\mathrm{d} \mathrm{t}}=\alpha \beta \mathrm{t}^{\beta-1} \mathrm{Q}(\mathrm{t})-\frac{\theta}{\mathrm{t}} \mathrm{Q}(\mathrm{t})-\lambda \mathrm{t}^{-\mathrm{p}} & \mu \leq \mathrm{t} \leq \mathrm{t}_{1} \\
\frac{\mathrm{dQ}(\mathrm{t})}{\mathrm{d} \mathrm{t}}=-\lambda \mathrm{t}^{-\mathrm{p}} & \mathrm{t}_{1} \leq \mathrm{t} \leq \mathrm{t}_{2} \\
\frac{\mathrm{dQ}(\mathrm{t})}{\mathrm{d} \mathrm{t}}=\mathrm{k}-\lambda \mathrm{t}^{-\mathrm{p}} & \mathrm{t}_{2} \leq \mathrm{t} \leq \mathrm{T}
\end{array}
$$

Using boundary conditions

$$
\mathrm{Q}(0)=0, \mathrm{Q}\left(\mathrm{t}_{1}\right)=0 \text { and } \mathrm{Q}(\mathrm{T})=0
$$

the solutions of the above differential equations (1), (2), (3) and (4) are given as

$$
\begin{aligned}
& \mathrm{Q}(\mathrm{t})=\mathrm{k} \mathrm{t}+\frac{\mathrm{k} \alpha \beta \mathrm{t}^{\beta+1}}{\beta+1}-\frac{\alpha \beta \lambda \mathrm{t}^{\beta-\mathrm{p}+1}}{(\beta-\mathrm{p}+1)(1-\mathrm{p})}-\frac{\lambda \mathrm{t}^{1-\mathrm{p}}}{1-\mathrm{p}} \\
& \mathrm{Q}(\mathrm{t})=\lambda\left[\begin{array}{l}
\left(\frac{\mathrm{t}_{1}^{\theta-\mathrm{p}+1}}{\theta-\mathrm{p}+1}-\frac{\alpha \mathrm{t}_{1}^{\theta-\mathrm{p}+\beta+1}}{\theta-\mathrm{p}+\beta+1}\right) \mathrm{t}^{-\theta}-\frac{\mathrm{t}^{1-\mathrm{p}}}{\theta-\mathrm{p}+1} \\
+\frac{\alpha \mathrm{t}^{\beta-\mathrm{p}+1}}{\theta+\beta-\mathrm{p}+1}+\frac{\alpha \mathrm{t}_{1}^{\theta-\mathrm{p}+1}}{\theta-\mathrm{p}+1} \mathrm{t}^{\beta-\theta}-\frac{\alpha \mathrm{t}^{\beta-\mathrm{p}+1}}{\theta-\mathrm{p}+1}
\end{array}\right] \\
& \mathrm{Q}(\mathrm{t})=\frac{\lambda}{1-\mathrm{p}}\left(\mathrm{t}_{1}^{1-\mathrm{p}}-\mathrm{t}^{1-\mathrm{p}}\right) \\
& \mathrm{Q}(\mathrm{t})=\left(\frac{\lambda \mathrm{T}^{1-\mathrm{p}}}{1-\mathrm{p}}-\mathrm{kT}\right)+\mathrm{kt}-\frac{\lambda \mathrm{t}^{1-\mathrm{p}}}{1-\mathrm{p}}
\end{aligned}
$$

Substituting $\mathrm{Q}\left(\mathrm{t}_{2}\right)=-\mathrm{S}_{2}$ in equations (7) and (8), we get

$$
\begin{aligned}
& -S_{2}=\frac{\lambda}{1-p}\left(t_{1}^{1-p}-t_{2}^{1-p}\right) \\
& -S_{2}=\left(\frac{\lambda T^{1-p}}{1-p}-k T\right)+\mathrm{kt}_{2}-\frac{\lambda t_{2}^{1-p}}{1-p}
\end{aligned}
$$

Eliminating $S_{2}$ from (9) and (10), we get

$$
\mathrm{t}_{2}=\mathrm{T}+\frac{1}{\mathrm{k}}\left(\frac{\lambda}{1-\mathrm{p}}\left(\mathrm{t}_{1}^{1-\mathrm{p}}-\mathrm{T}^{1-\mathrm{p}}\right)\right)
$$

Thus $t_{2}$ can be expressed in terms of $t_{1}$ and $T$.

\section{Cost Components}

On the basis of the assumptions and description of the model, the total cost consists of the following cost components:

\subsection{Operating Cost (OC)}

The operating cost over the period $[0, \mathrm{~T}]$ is

$$
\mathrm{OC}=\mathrm{A}
$$




\subsection{Inventory Holding Cost (IHC)}

The holding cost for carrying inventory over the period $\left[0, t_{1}\right]$ is

$$
\begin{aligned}
& \mathrm{IHC}=\int_{0}^{\mu}(\mathrm{h}+\mathrm{rt}) \mathrm{Q}(\mathrm{t}) \mathrm{dt}+\int_{\mu}^{\mathrm{t}_{1}}(\mathrm{~h}+\mathrm{rt}) \mathrm{Q}(\mathrm{t}) \mathrm{dt}
\end{aligned}
$$

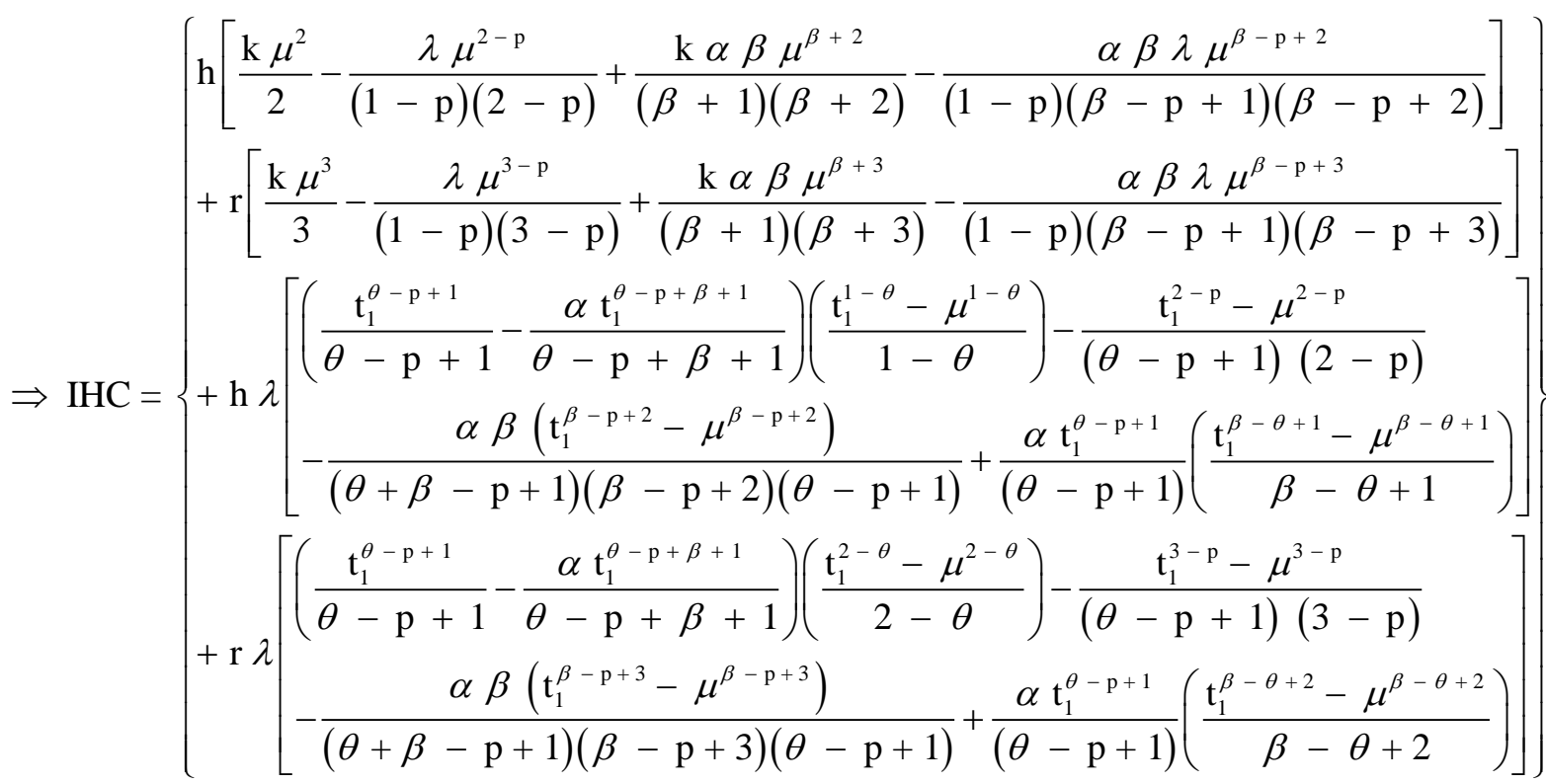

\subsection{Deterioration Cost (DC)}

The deterioration cost during the period $\left[\mu, t_{1}\right]$ is

$$
\begin{aligned}
\mathrm{DC}=\mathrm{C}_{\mathrm{d}} \int_{\mu}^{\mathrm{t}_{1}} \frac{\theta}{\mathrm{t}} \mathrm{Q}(\mathrm{t}) \mathrm{dt} \\
\Rightarrow \mathrm{DC}=\lambda \theta\left[\begin{array}{c}
\left(\frac{\mathrm{t}_{1}^{\theta-\mathrm{p}+1}}{\theta-\mathrm{p}+1}-\frac{\alpha \mathrm{t}_{1}^{\theta-\mathrm{p}+\beta+1}}{\theta-\mathrm{p}+\beta+1}\right)\left(\frac{\mathrm{t}_{1}^{-\theta}-\mu^{-\theta}}{-\theta}\right)-\frac{\mathrm{t}_{1}^{1-\mathrm{p}}-\mu^{1-\mathrm{p}}}{(\theta-\mathrm{p}+1)(1-\mathrm{p})} \\
-\frac{\alpha \beta\left(\mathrm{t}_{1}^{\beta-\mathrm{p}+1}-\mu^{\beta-\mathrm{p}+1}\right)}{(\theta+\beta-\mathrm{p}+1)(\beta-\mathrm{p}+1)(\theta-\mathrm{p}+1)}+\frac{\alpha \mathrm{t}_{1}^{\theta-\mathrm{p}+1}}{(\theta-\mathrm{p}+1)}\left(\frac{\mathrm{t}_{1}^{\beta-\theta}-\mu^{\beta-\theta}}{\beta-\theta}\right)
\end{array}\right] \ldots
\end{aligned}
$$

\subsection{Production Cost (PC)}

The production cost per cycle is

$$
\mathrm{PC}=\mathrm{C}_{\mathrm{p}} \mathrm{k}\left(\mu+\mathrm{T}-\mathrm{t}_{2}\right)
$$

\subsection{Shortage Cost (SC)}

The shortage cost during the period $\left[\mathrm{t}_{1}, \mathrm{~T}\right]$ is

$$
S C=-C_{S}\left[\int_{t_{1}}^{t_{2}} Q(t) d t+\int_{t_{2}}^{T} Q(t) d t\right]
$$




$$
\Rightarrow \mathrm{SC}=-\mathrm{C}_{\mathrm{s}}\left[\begin{array}{l}
\frac{\lambda}{1-\mathrm{p}}\left(\mathrm{t}_{1}^{1-\mathrm{p}}\left(\mathrm{t}_{2}-\mathrm{t}_{1}\right)-\left(\frac{\mathrm{t}_{2}^{2-\mathrm{p}}-\mathrm{t}_{1}^{2-\mathrm{p}}}{2-\mathrm{p}}\right)\right)+\left(\frac{\lambda \mathrm{T}^{1-\mathrm{p}}}{1-\mathrm{p}}-\mathrm{kT}\right)\left(\mathrm{T}-\mathrm{t}_{2}\right) \\
+\mathrm{k}\left(\frac{\mathrm{T}^{2}-\mathrm{t}_{2}^{2}}{2}\right)-\frac{\lambda\left(\mathrm{T}^{2-\mathrm{p}}-\mathrm{t}_{2}^{2-\mathrm{p}}\right)}{(1-\mathrm{p})(2-\mathrm{p})}
\end{array}\right]
$$

\subsection{Amelioration Cost (AMC)}

The amelioration cost over the period $\left[0, \mathrm{t}_{1}\right]$ is

$$
\begin{aligned}
& \mathrm{AMC}=\mathrm{C}_{\mathrm{a}}\left[\int_{0}^{\mu} \alpha \beta \mathrm{t}^{\beta-1} \mathrm{Q}(\mathrm{t}) \mathrm{d} \mathrm{t}+\int_{\mu}^{\mathrm{t}_{1}} \alpha \beta \mathrm{t}^{\beta-1} \mathrm{Q}(\mathrm{t}) \mathrm{d} \mathrm{t}\right] \\
& \Rightarrow \mathrm{AMC}=\mathrm{C}_{\mathrm{a}} \alpha \beta\left[\begin{array}{l}
\frac{\mathrm{k} \mu^{\beta+1}}{\beta+1}-\frac{\lambda \mu^{\beta-\mathrm{p}+1}}{(1-\mathrm{p})(\beta-\mathrm{p}+1)}+\frac{\lambda \mathrm{t}_{1}^{\theta-\mathrm{p}+1}\left(\mathrm{t}_{1}^{\beta-\theta}-\mu^{\beta-\theta}\right)}{(\theta-\mathrm{p}+1)(\beta-\theta)} \\
-\frac{\lambda\left(\mathrm{t}_{1}^{\beta-\mathrm{p}+1}-\mu^{\beta-\mathrm{p}+1}\right)}{(\theta-\mathrm{p}+1)(\beta-\mathrm{p}+1)}
\end{array}\right]
\end{aligned}
$$

\section{Total Cost (TC)}

Taking the relevant costs mentioned above, the total average cost of the system is given by

$$
\begin{aligned}
& \mathrm{TC}=\frac{1}{\mathrm{~T}}[\mathrm{OC}+\mathrm{IHC}+\mathrm{DC}+\mathrm{PC}+\mathrm{SC}+\mathrm{AMC}]
\end{aligned}
$$

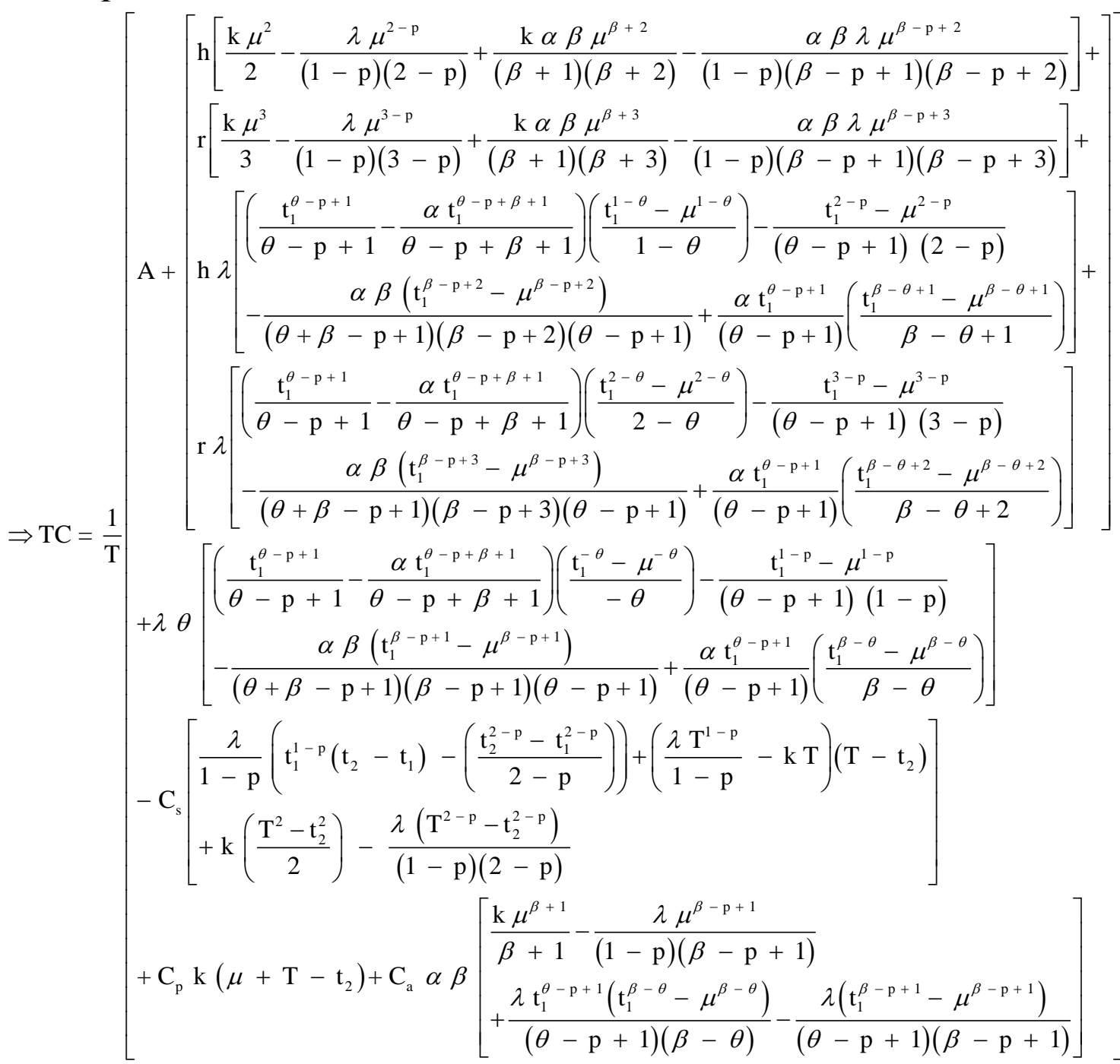


Production Inventory System under Weibull Amelioration, Pareto Deterioration and Exponentially Time Based Demand under Fully Backlogged Shortages

Our objective is to determine optimum values $\mu^{*}, t_{1}^{*}$ and $T^{*}$ of $\mu, t_{1}$ and $T$ respectively so that $\mathrm{TC}$ is minimum. Note that values $\mu^{*}, t_{1}^{*}$ and $T^{*}$ can be obtained by solving the equations

$\frac{\partial(\mathrm{TC})}{\partial \mu}=0, \frac{\partial(\mathrm{TC})}{\partial \mathrm{t}_{1}}=0 \& \frac{\partial(\mathrm{TC})}{\partial \mathrm{T}}=0$

with sufficient conditions

$\left.\begin{array}{l}\left|\begin{array}{lll}\frac{\partial^{2} \mathrm{TC}}{\partial \mu^{2}} & \frac{\partial^{2} \mathrm{TC}}{\partial \mu \partial \mathrm{t}_{1}} & \frac{\partial^{2} \mathrm{TC}}{\partial \mu \partial \mathrm{T}} \\ \frac{\partial^{2} \mathrm{TC}}{\partial \mathrm{t}_{1} \partial \mu} & \frac{\partial^{2} \mathrm{TC}}{\partial \mathrm{t}_{1}^{2}} & \frac{\partial^{2} \mathrm{TC}}{\partial \mathrm{t}_{1} \partial \mathrm{T}} \\ \frac{\partial^{2} \mathrm{TC}}{\partial \mathrm{T} \partial \mu} & \frac{\partial^{2} \mathrm{TC}}{\partial \mathrm{T} \partial \mathrm{t}_{1}} & \frac{\partial^{2} \mathrm{TC}}{\partial \mathrm{T}^{2}}\end{array}\right|_{\mu=\mu^{*}, \mathrm{t}_{1}=\mathrm{t}_{1}^{*}, \mathrm{~T}=\mathrm{T}^{*}}>0,\left|\begin{array}{cc}\frac{\partial^{2} \mathrm{TC}}{\partial \mu^{2}} & \frac{\partial^{2} \mathrm{TC}}{\partial \mu \partial \mathrm{t}_{1}} \\ \frac{\partial^{2} \mathrm{TC}}{\partial \mathrm{t}_{1} \partial \mu} & \frac{\partial^{2} \mathrm{TC}}{\partial \mathrm{t}_{1}^{2}}\end{array}\right|_{\mu=\mu^{*}, \mathrm{t}_{1}=\mathrm{t}_{1}^{*}, \mathrm{~T}=\mathrm{T}^{*}}>0 \\ \left.\& \frac{\partial^{2} \mathrm{TC}}{\partial \mu^{2}}\right|_{\mu=\mu^{*}, \mathrm{t}_{1}=\mathrm{t}_{1}^{*}, \mathrm{~T}=\mathrm{T}^{*}}>0\end{array}\right\} . .$.

The optimal solution of the equations in (19) can be obtained by using appropriate software.

\section{NUMERICAL EXAMPLE}

We consider the following numerical example to illustrate the above inventory model. We take the values of the parameters $A=350, p=0.2, h=7, r=3, C_{p}=30, \alpha=0.0001, C_{d}=13, \beta=7, \lambda=10, k=$ $25, \mathrm{C}_{\mathrm{s}}=24, \theta=4$ and $\mathrm{C}_{\mathrm{a}}=12$ (with appropriate units of measurement). We obtain the values $\mu=$ 0.9463 units, $\mathrm{t}_{1}=1.4480$ units, $\mathrm{T}=4.0082$ units and total cost $\mathrm{TC}=581.00$ units by using appropriate software.

\section{Sensitivity ANALysis}

Sensitivity analysis is very important technique to identify the effect on optimal solution of the model by changing its parameter values. In this section, we study the sensitivity of total cost TC per time unit with respect to the changes in the values of the parameters $A, p, h, r, C_{p}, \alpha, C_{d}, \beta, \lambda, k, C_{s}, \theta$ and $\mathrm{C}_{\mathrm{a}}$

This analysis is performed by considering $10 \%$ and $20 \%$ increase and decrease in each one of the above parameters keeping all other remaining parameter as fixed. The results are presented in the Table below. The last column of the table shows the \% change in TC as compared to the original solution corresponding to the change in parameters values, taken one by one.

\section{Sensitivity Analysis}

Table: Sensitivity analysis of parameters considered in defining inventory model

\begin{tabular}{|c|c|c|c|c|c|c|}
\hline Parameter & $\begin{array}{c}\text { \% } \\
\text { change }\end{array}$ & $\boldsymbol{\mu}$ & $\mathbf{t}_{\mathbf{1}}$ & $\mathbf{T}$ & TC & \% change in TC \\
\hline \multirow{3}{*}{$\mathbf{A}$} & -20 & 0.83175589 & 1.266598147 & 3.629045911 & 562.6778426 & -3.154367106 \\
\cline { 2 - 7 } & -10 & 0.89102353 & 1.360275867 & 3.824622008 & 572.0686178 & -1.538068228 \\
\cline { 2 - 7 } & +10 & 0.998302145 & 1.530564752 & 4.181725486 & 589.5515557 & 1.471018078 \\
\cline { 2 - 7 } & +20 & 1.047335814 & 1.60871594 & 4.346500791 & 597.759319 & 2.883702162 \\
\hline \multirow{3}{*}{$\mathbf{p}$} & -20 & 0.859701786 & 1.31110523 & 3.769610753 & 589.1869838 & 1.408269563 \\
\cline { 2 - 7 } & -10 & 0.903711768 & 1.380337631 & 3.888256076 & 585.1925571 & 0.720766421 \\
\cline { 2 - 7 } & +10 & 0.987897045 & 1.514481235 & 4.130136151 & 576.6313298 & -0.75275432 \\
\cline { 2 - 7 } & +20 & 1.028580335 & 1.580204783 & 4.254440932 & 572.0774381 & -1.53655011 \\
\hline \multirow{2}{*}{$\mathbf{h}$} & -20 & 1.00621809 & 1.54390374 & 4.107218692 & 578.3496366 & -0.457006928 \\
\cline { 2 - 7 } & -10 & 0.97520929 & 1.494188491 & 4.055958443 & 579.7142188 & -0.222141037 \\
\hline
\end{tabular}


Ankit Bhojak \& U. B. Gothi

\begin{tabular}{|c|c|c|c|c|c|c|}
\hline & +10 & 0.919440181 & 1.404948498 & 3.963745791 & 582.2279515 & 0.210511559 \\
\hline & +20 & 0.894291303 & 1.364778613 & 3.922158 & 583.3891243 & 0.410367515 \\
\hline \multirow{4}{*}{$\mathbf{r}$} & -20 & 0.97836117 & 1.499141683 & 4.065402182 & 580.1096796 & -0.154076074 \\
\hline & -10 & 0.96175812 & 1.472597431 & 4.03572866 & 580.5675553 & -0.075268529 \\
\hline & +10 & 0.93199116 & 1.425056323 & 3.982674506 & 581.4235578 & 0.072062863 \\
\hline & +20 & 0.918550719 & 1.403611046 & 3.95878191 & 581.825281 & 0.141205688 \\
\hline \multirow{4}{*}{$\mathrm{C}_{\mathrm{p}}$} & -20 & 1.037274297 & 1.540212251 & 3.939419913 & 512.7097178 & -11.75466074 \\
\hline & -10 & 0.990215601 & 1.49328101 & 3.975277894 & 547.1746475 & -5.822708784 \\
\hline & +10 & 0.905757256 & 1.404892347 & 4.039542864 & 614.265725 & 5.724712076 \\
\hline & +20 & 0.868429347 & 1.364413278 & 4.07016762 & 647.014448 & 11.3612781 \\
\hline \multirow{4}{*}{$\alpha$} & -20 & 0.946241944 & 1.447780841 & 4.007998621 & 581.0059841 & 0.000191915 \\
\hline & -10 & 0.946296945 & 1.447882528 & 4.008122461 & 581.0054268 & 0.000095995 \\
\hline & +10 & 0.946407202 & 1.448086356 & 4.008370699 & 581.0043233 & -0.00009393 \\
\hline & +20 & 0.946462458 & 1.448188499 & 4.0084951 & 581.0037523 & -0.00019222 \\
\hline \multirow{4}{*}{$\mathbf{C}_{\mathrm{d}}$} & -20 & 0.958475485 & 1.51802264 & 4.04577743 & 576.0678279 & -0.849741786 \\
\hline & -10 & 0.9529651 & 1.482227301 & 4.027633266 & 578.4065998 & -0.447202661 \\
\hline & +10 & 0.939019323 & 1.415379682 & 3.988252259 & 583.6186477 & 0.449872076 \\
\hline & +20 & 0.93123837 & 1.384425497 & 3.968080257 & 585.9711122 & 0.854767899 \\
\hline \multirow{4}{*}{$\boldsymbol{\beta}$} & -20 & 0.945924244 & 1.447251212 & 4.007352972 & 581.0088104 & 0.000678365 \\
\hline & -10 & 0.946095172 & 1.447546686 & 4.007712133 & 581.0071054 & 0.000384908 \\
\hline & +10 & 0.946727253 & 1.448617365 & 4.009021841 & 581.0019521 & -0.000502048 \\
\hline & +20 & 0.947266002 & 1.449519586 & 4.01012983 & 580.998157 & -0.00115526 \\
\hline \multirow{4}{*}{$\lambda$} & -20 & 0.711698697 & 1.126870535 & 3.942689058 & 526.8454182 & -9.321686228 \\
\hline & -10 & 0.831906411 & 1.294211397 & 3.975372781 & 555.6323446 & -4.367007193 \\
\hline & +10 & 1.056702954 & 1.591573486 & 4.042661113 & 603.4029699 & 3.855062498 \\
\hline & +20 & 1.1646265 & 1.728059591 & 4.079945996 & 623.1660866 & 7.256603129 \\
\hline \multirow{4}{*}{$\mathbf{k}$} & -20 & 1.342361729 & 1.984224462 & 4.476893386 & 522.035381 & -10.14956865 \\
\hline & -10 & 1.12600564 & 1.696279152 & 4.228863153 & 553.6095199 & -4.71516688 \\
\hline & +10 & 0.792027081 & 1.227334262 & 3.802834813 & 604.8840884 & 4.109986095 \\
\hline & +20 & 0.6571643 & 1.028832758 & 3.607387127 & 625.7092291 & 7.694317618 \\
\hline \multirow{4}{*}{$\mathbf{C}_{\mathrm{s}}$} & -20 & 0.860126072 & 1.311404129 & 4.296908106 & 555.2920885 & -4.42557059 \\
\hline & -10 & 0.90565684 & 1.383448927 & 4.137856415 & 568.9304429 & -2.078197073 \\
\hline & +10 & 0.982935162 & 1.50611407 & 3.900384517 & 591.7867648 & 1.855732415 \\
\hline & +20 & 1.015999855 & 1.558748329 & 3.809082029 & 602.2973344 & 3.664765379 \\
\hline \multirow{4}{*}{$\boldsymbol{\theta}$} & -20 & 0.980314302 & 1.636660992 & 4.139036945 & 568.0747475 & -2.225475592 \\
\hline & -10 & 0.964425575 & 1.53543882 & 4.070841421 & 575.1847286 & -1.001736951 \\
\hline & +10 & 0.927502049 & 1.372108242 & 3.95131385 & 585.8344683 & 0.831249364 \\
\hline & +20 & 0.908736406 & 1.30600132 & 3.899810838 & 589.8909184 & 1.529427684 \\
\hline \multirow{4}{*}{$\mathbf{C}_{\mathbf{a}}$} & -20 & 0.946575248 & 1.44833933 & 4.008676399 & 581.0026323 & -0.000384979 \\
\hline & -10 & 0.946463543 & 1.448161694 & 4.008461255 & 581.0037512 & -0.000192402 \\
\hline & +10 & 0.946240711 & 1.447807344 & 4.008032093 & 581.0059859 & 0.000192226 \\
\hline & +20 & 0.946129582 & 1.447630627 & 4.007818073 & 581.0071017 & 0.000384278 \\
\hline
\end{tabular}




\section{Graphical Presentation}

Graphical presentation of the above sensitivity analysis is shown in Fig. 2.

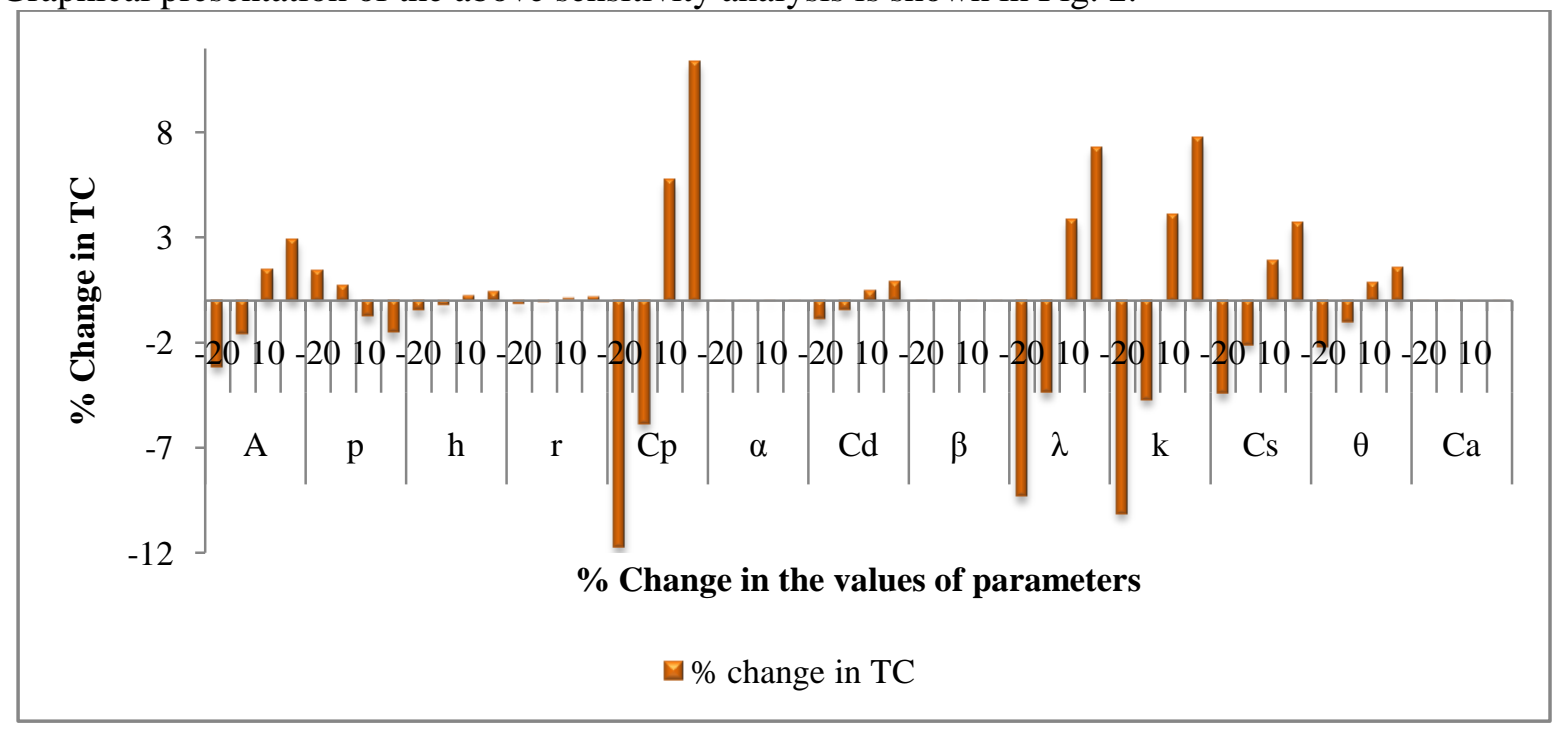

Fig. 2. Graphical presentation of the above sensitivity analysis

\section{CONCLUSION}

From the above sensitivity analysis we may conclude that the total cost TC per time unit is highly sensitive to the changes in the values of the parameters $C_{p}, k$ and $\lambda$; moderately sensitive to the changes in the values of the parameters $C_{s}, A$ and $\theta$ and less sensitive to the changes in the values of the parameters $p, C_{d}, h, r, \alpha, \beta$ and $C_{a}$.

Moreover, It can also be observed from Fig. 2. that there is an opposite change in total cost TC per time unit for parameters $\mathrm{p}, \alpha$ and $\beta$ where as simultaneous change can be found in total cost TC per time unit for the remaining parameters $\mathrm{C}_{\mathrm{p}}, \mathrm{k}, \lambda, \mathrm{C}_{\mathrm{s}}, \mathrm{A}, \theta, \mathrm{C}_{\mathrm{d}}, \mathrm{h}, \mathrm{r}$ and $\mathrm{C}_{\mathrm{a}}$.

\section{REFERENCES}

[1] Ankit Bhojak and U. B. Gothi, "EPQ Model with Time Dependent IHC and Weibull Distributed Deterioration under Shortages," International Journal of Innovative Research in Science, Engineering and Technology, pp. 10748-10757, (2015).

[2] Ankit Bhojak and U. B. Gothi, "Inventory Models for Ameliorating and Deteriorating Items with Time Dependent Demand and IHC," International Journal of Management and Social Science Research Review, Vol.1, Issue.2, pp. 85 - 98, (2016).

[3] H. Hwang, "A stochastic set-covering location model for both ameliorating and deteriroating items," Computers and Industrial Engineering, 46, pp. 313-319,(2004).

[4] H. C. Chang, "A Note on the EPQ Model with Shortages and Variable Lead Time," Information and Management Sciences Vol. 15, No.1, pp. 61-67, (2004).

[5] Rao, K. S., Begum, K. J., and Murty, M. V., "Optimal ordering policies of inventory model for deteriorating items having generalized Pareto lifetime," CURRENT SCIENCE-BANGALORE-, 93(10), pp. 1407-1411, (2007).

[6] Srichandan Mishra, L.K.Raju, U.K.Misra and G.Misra, "Optimal Control of An Inventory System with Variable Demand \& Ameliorating / Deteriorating Items," Asian Journal of Current Engineering and Maths, p. 154 - 157, (2012).

[7] Vijayalakshmi. G, Srinivasa Rao. K and Nirupama Devi. K, "Inventory Model for Deteriorating Items Having Two Component Mixture of Pareto Lifetime and Selling Price Dependent Demand," International Journal of Scientific \& Engineering Research, Volume 5, Issue 7, July, pp. 254-262, (2014). 
[8] Kapil Kumar Bansal and Navin Ahalawat, "Integrated Inventory Models for Decaying Items with Exponential Demand under Inflation," International Journal of Soft Computing and Engineering (IJSCE) Volume-2, Issue-3, (2012).

[9] U.B. Gothi and Devyani A. Chatterji, "EPQ model for Imperfect Quality Items Under Constant Demand Rate and Varying IHC," Sankhya Vignan NSV11, pp. 7 - 19, (2015).

[10] Madhu Jain, G.C. Sharma and Shalini Rathore, "Economic production quantity models with Shortage, price and stock-dependent Demand for deteriorating items," IJE Transactions Vol. 20, No 2, pp. 159-166, (2007).

[11] R. Shamsi, A. Haji, S. Shadrokh and F. Nourbakhsh, "Economic Production Quantity in Reworkable Production Systems with Inspection Errors, Scraps and Backlogging," Journal of Industrial and Systems Engineering Vol. 3, No. 3, pp. 170-188, (2009).

[12] I. Moon, B.C. Giri and B. Ko, "Economic order quantity models for ameliorating/deteriorating items under inflation and time discounting," European Journal of Operations Research, 162, pp. 773-785, (2005).

[13] R. Babu Krishnaraj and K. Ramasamy, "An Inventory Model With Power Demand Pattern, Weibull Distribution Deterioration and without Shortages," The Bulletin of Society for Mathematical Services and Standards, Vol.2, pp. 33-37, (2012).

[14] S.T. Law and H.M. Wee, "An integrated production inventory for ameliorating and deteriorating items taking account of time discounting," Mathematical and Computer Modelling, 43, pp. 673-685, (2006).

[15] Kawale S.V. and Bansode P.B., "An EPQ model using Weibull deterioration for deterioration item with time varying holding cost," International Journal of Science, Engineering and Technology Research, vol.1, issue 4, pp. 29-33, (2012).

[16] Kirtan Parmar and U. B. Gothi, "An EPQ model of deteriorating items using three parameter Weibull distribution with constant production rate and time varying holding cost," International Journal of Science, Engineering and Technology Research, Vol. 4, p. 409 - 416, (2015).

[17] Singh, N., Vaish, B., and Singh, S. R., "An EOQ Model with Pareto Distribution for Deterioration, Trapezodial Type Demand and Backlogging under Trade Credit Policy," The IUP Journal of Computational Mathematics, 3(4), pp. 30-53, (2010).

[18] Ruxian Li, Hongjie Lan and John R. Mawhinney, "A Review on Deteriorating Inventory Study," J.Service Science \& Management, 3, pp. 117-129, (2010).

[19] Hui- Ming Wee, Wan-Tsu Wang and Po-Chung Yang, "A production quantity model for imperfect quality items with shprtage and screening constraint," International journal of Production research. Vol.51, No.6, pp. 1869-1884, (2013).

[20] Shirajul Islam Ukil, Mohammad Ekramol Islam and Md. Sharif Uddin, "A Production Inventory Model of Power Demand and Constant Production Rate Where the Products Have Finite ShelfLife," Journal of Service Science and Management, pp. 874-885, (2015).

[21] Chun-Hsiung Lan, Yen-Chieh Yu, Robert H.-J. Lin, Cheng-Tan Tung, Chih-Pin Yen and Peter Shaohua Deng, "A Note on the Improved Algebric Method for the EPQ Model with Stochastic Lead Time," Information and Management Sciences Vol. 18, No.1, pp. 91-96, (2007).

[22] U. B. Gothi, Devyani A. Chatterji and Kirtan C. Parmar, "An Integrated Inventory Model with Exponential Amelioration and Two Parameter Weibull Deterioration," Journal of Statistics and Mathematical Engineering, MAT Journals, Vol.2, No. 2, (2016).

[23] U.B. Gothi and Ankit Bhojak, "Inventory Model with Pareto Type - I Distributed Deterioration Under Power Pattern Demand and Partial Backlogging," Sankhya Vignan, NSV 12, June, No. 1 Issue, (2016). 


\section{AUTHORS' BIOGRAPHY}

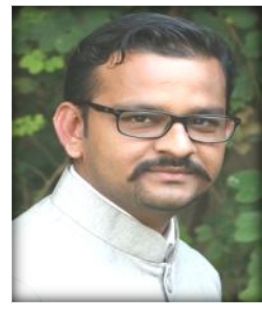

Ankit Bhojak, is an Assistant Professor of Statistics in GLS (Smt. M.R. Parikh) Institute of Commerce, GLS University, Ahmedabad and a Research scholar in Statistics at Gujarat University. Owning the credit of authoring books for graduate programme, he is an educator with intense motive of sharing his wide knowledge in the academic arena. He has been teaching Statistics and Mathematics to elementary, graduation and post gradation students of science and commerce. He has also contributed articles to various journals of Statistics and research.

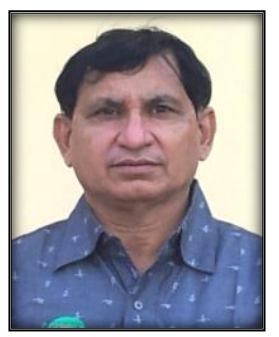

A recognised guide at Gujarat University, Ahmedabad, Dr. Ushakar B. Gothi is the Head and Associate Professor of Statistics at St. Xavier's College (Autonomous), Gujarat University, Ahmedabad who has tremendous teaching experience of around 37 years at under graduate and post graduate level. He has been member of various boards and committees of Gujarat Education Board as well as Gujarat University. He is also an editorial member of one of the reputed international journals. He also contributed numerous research articles in National and International Journals. 